\title{
Search for High-Energy Neutrino Emission from Fast Radio Bursts
}

\author{
The IceCube Collaboration ${ }^{\dagger}$ \\ ${ }^{\dagger}$ http://icecube.wisc.edu/collaboration/authors/icrc17_icecube \\ E-mail: dxueicecube.wisc.edu
}

\begin{abstract}
Fast radio bursts (FRBs) are irregular millisecond radio outbursts that are thought to be of astrophysical origin. The first FRB was discovered by the Parkes Radio Telescope in 2007. During this search period from May 2010 to May 2016, a total of 29 FRBs with 13 unique locations (FRB 121102 has repeated 17 times) have been observed, with addition of the Green Bank and Arecibo radio telescopes to the discovery facilities. Although the nature of the FRBs is still largely unknown, the high dispersion measures of the FRBs indicate that they are most likely originating from extragalactic sources. A multitude of models have been proposed to explain the FRB phenomena, most of which involve strong magnetic fields and are of leptonic nature. Currently, there are no concrete models predicting high-energy neutrinos from FRBs, while in principle a strongly magnetized environment such as that from a magnetar could produce short radio bursts due to the volatility of the magnetic fields, and having hadronic processes present at the same time. We will present the results from a recent search for high-energy neutrinos coincident spatially and temporally with FRBs in 6 years of IceCube data.
\end{abstract}

Corresponding authors: ${ }^{1}$ Sam Fahey, ${ }^{1}$ Justin Vandenbroucke and ${ }^{1}$ Donglian $\mathrm{Xu}^{*}$

${ }^{1}$ Wisconsin IceCube Particle Astrophysics Center and Department of Physics, University of Wisconsin, Madison, WI 53706, USA

35th International Cosmic Ray Conference - ICRC2017-

10-20 July, 2017

Bexco, Busan, Korea

${ }^{*}$ Speaker. 


\section{Introduction}

Fast radio bursts (FRBs) are a new category of bright, broadband, transient astrophysical radio outbursts which typically last a few milliseconds. The first FRB, FRB 010724, was discovered in 2007 in the archival data collected on January 7, 2001 by the Parkes Radio Telescope [1]. More FRBs were found later by several different instruments including the Green Bank Telescope and the Arecibo Telescope, eliminating the instrumental origin hypothesis [2,3]. To date, a total of 23 FRBs with unique locations have been detected, of which only FRB 121102 has been observed to repeat by multiple instruments $[4,5]$. The nature of FRB progenitors is largely unknown. The sources are thought to be extremely compact ( $\sim$ hundreds of kilometers) due to causality. The majority of FRBs are detected at high Galactic latitudes due to limited telescope coverage bias, with much higher dispersion measures (DM) than expected from a Galactic contribution, indicating extragalactic origin. Despite the limited number of detected bursts, the all-sky occurrence rate for FRBs is estimated to be a few thousand per day, consistent with the supernova rate[3, 6]. There exists a multitude of models for FRB progenitors, the majority of which involve strong magnetic fields and leptonic process. Some models associate FRBs with cataclysmic events such as dying stars [7], neutron star mergers [8] or evaporating black holes [9]. The repeated FRB 121102, however, refuted the cataclysmic cause for this burst. Because of its repeating nature, a multiwavelength follow-up campaign was able to locate this burst's origin to a dwarf galaxy at a redshift of $z=0.19$, at a distance of $\sim 1 \mathrm{Gpc}$ [5]. No convincing models exist to explain the repeating burst, although a millisecond magnetar has been recently proposed [10]. It is unclear whether the repeating burst is typical for the FRB population or not, as no other FRBs have been localized to their host galaxies. Multi-wavelength and multi-messenger follow ups would provide additional information and help shed light on the FRB progenitor puzzle. One such follow up study would be a search for high-energy neutrino counterparts with the IceCube neutrino observatory.

IceCube is a cubic-kilometer neutrino detector installed in the ice at the geographic South Pole [11] between depths of $1450 \mathrm{~m}$ and $2450 \mathrm{~m}$, completed in 2010. Reconstruction of the direction, energy and to some extent the flavor of the neutrinos relies on the optical detection of Cherenkov radiation emitted by charged particles produced in the interactions of neutrinos in the surrounding ice or the nearby bedrock. IceCube collects data from the whole sky with greater than 99\% up time per year, enabling real-time alerts to other instruments and archival data follow ups on interesting signals from other detectors.

\section{Data Samples}

The data samples used in this analysis were based on track-like muon neutrino candidate events selected from previous analyses in search for coincident neutrinos with gamma ray bursts $[12,13]$. Specifically, the first year sample from the northern hemisphere (declination $>-5^{\circ}$ ) was from [12] and the rest of both northern and southern hemisphere (declination $<-5^{\circ}$ ) samples were from [13]. The data taking period for these data samples was from May 31, 2010 to May 20, 2016. During this time, there were 4 unique FRBs detected in the northern hemisphere, namely FRB 110523 (Green Bank Telescope), FRB 110703 (Parkes Telescope), FRB 121102 (Arecibo Telescope) and FRB 130628 (Parkes Telescope). Only 17 of the repeated bursts from FRB 121102 are included in 

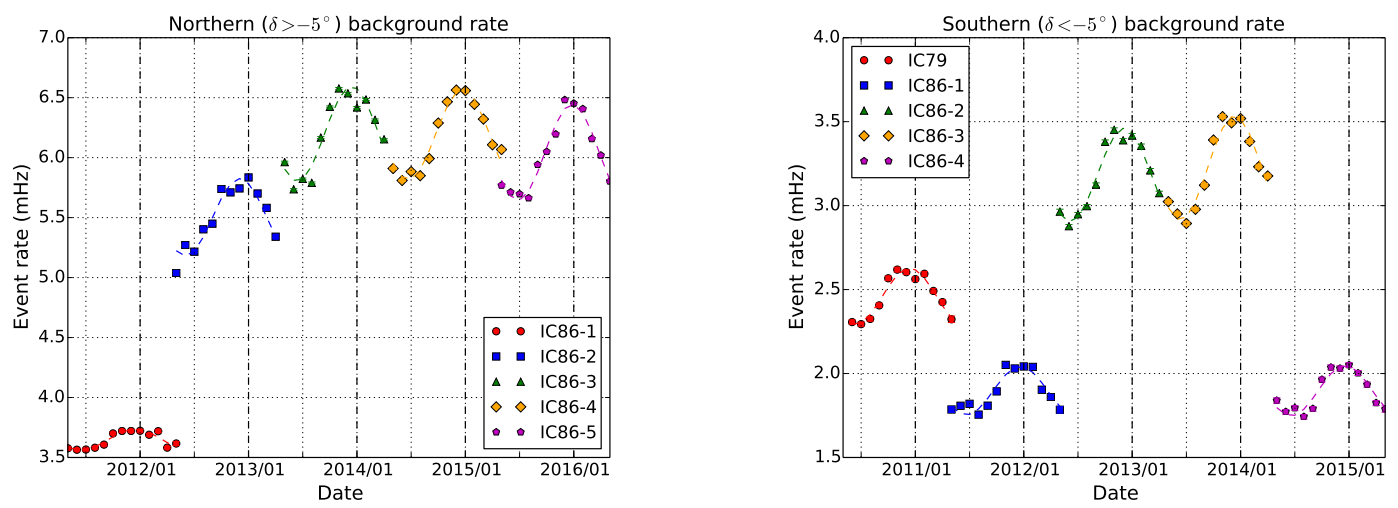

Figure 1: Left: northern hemisphere event distributions. The label "IC86-1" indicates the first year when IceCube was taking data with the full 86 string configuration in 2011. Right: southern hemisphere event distributions. The label "IC79" denotes the year when IceCube was taking data with the 79 string configuration in 2010.

this analysis and treated as unique bursts in space and time. In the southern hemisphere, there are 9 unique FRBs, all of which were detected by the Parkes Telescope. The northern event samples consist primarily of atmospheric muon neutrinos because the Earth acts as a filter for cosmic-ray induced atmospheric muons. On the other hand, the southern samples are dominated by down-going atmospheric muons despite a higher energy threshold in the event selection to reduce the enormous atmospheric muon background. As shown in Fig. 1, the first year northern sample has an overall event rate $\sim 3.5 \mathrm{mHz}$, while the rest of the later four year samples have a rate $\sim 6 \mathrm{mHz}$. The southern samples have event rates $\sim 2-3 \mathrm{mHz}$. The year to year event rate variation was a combined effect of initial event selections treating each year of the IceCube data sample independently due to frequent filter and data processing scheme updates in the early years of IceCube operation, and optimization to the per year sensitivity which was dependent on the amount of selected GRBs. The annual seasonal variations for both atmospheric muon and neutrino rates are thought to be caused by a combination of temperature and column density of air nuclei changes in the atmosphere over different seasons. The seasonal variations on event rates were taken into account when constructing background ensembles.

The events that are within \pm 2 days from any FRB burst time are defined as the on time samples, which will be used to search for correlations once the analysis methods are finalized (see Section 3). The rest of the events are called off time samples, and were used in background modeling.

\section{Analysis Methods}

This analysis is designed to search for spatial and temporal correlation between observed fast radio bursts and neutrinos detected by IceCube. An unbinned maximum likelihood method is employed in the search, during which a Poissonian time distribution is assumed. The likelihood for observing $N$ events with properties $\left\{x_{i}\right\}$ for $\left(n_{s}+n_{b}\right)$ expected number of events is 


$$
L\left(N,\left\{x_{i}\right\} ; n_{s}+n_{b}\right)=\frac{\left(n_{s}+n_{b}\right)^{N}}{N !} \cdot \exp \left(-\left(n_{s}+n_{b}\right)\right) \cdot \prod_{i=1}^{N} \frac{n_{s} S\left(x_{i}\right)+n_{b} B\left(x_{i}\right)}{n_{s}+n_{b}}
$$

where $n_{s}$ and $n_{b}$ are the expected number of signal and background events, and $S\left(x_{i}\right)$ and $B\left(x_{i}\right)$ are the signal and background PDFs. The piecewise contributions to the signal and background PDFs are only spatial and temporal, so no energy dependent PDF is included. A generic test statistic used in this analysis is defined as the logarithmic ratio of the alternative hypothesis and the null hypothesis, $T S:=\ln L\left(N,\left\{x_{i}\right\} ; n_{s}+n_{b}\right) / L_{0}\left(N,\left\{x_{i}\right\} ; n_{b}\right)$, which can be simplified as

$$
T S:=-\hat{n}_{s}+\sum_{i=1}^{N} \ln \left(1+\frac{\hat{n}_{s} S_{i}}{\left\langle n_{b}\right\rangle B_{i}}\right)
$$

To find the most probable number of signal-like events $n_{s}$ from $N$ observed events, one needs to maximize the likelihood function $L$ with respect to $\hat{n}_{s}$, which is equivalent to maximizing the test statistic $T S$ because $L_{0}$ is a constant.
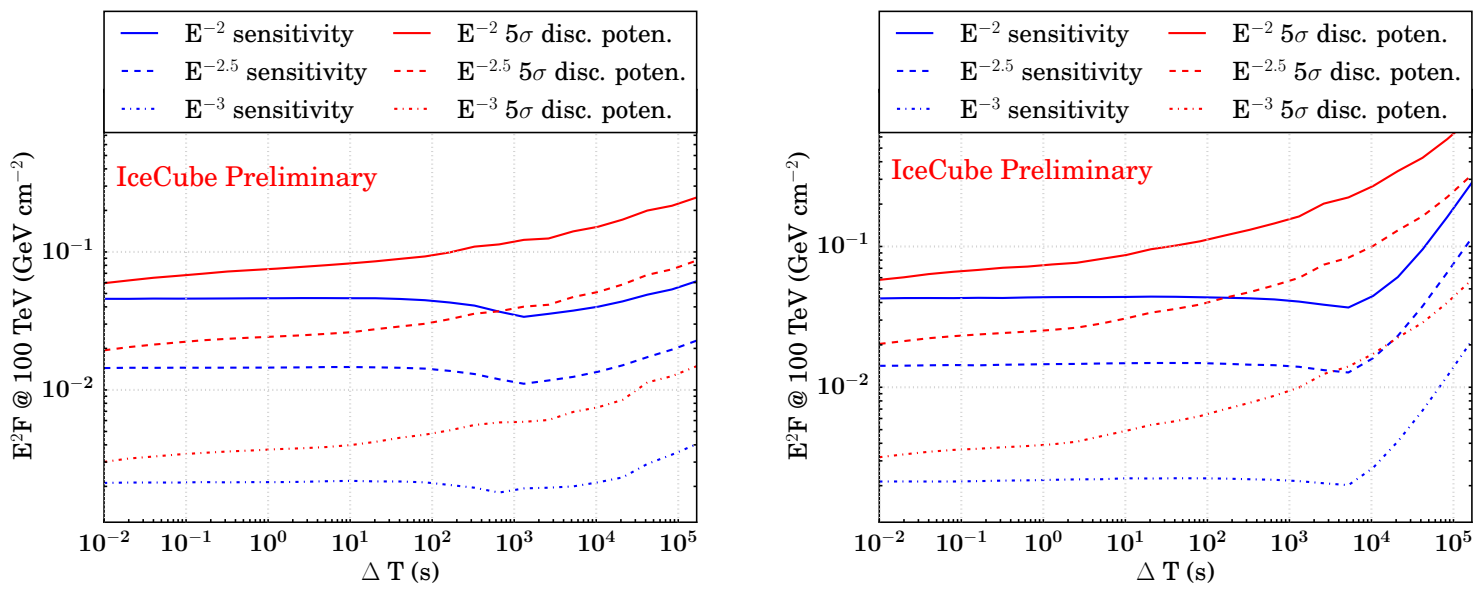

Figure 2: Left: the $90 \%$ confidence level pre-trial sensitivity and $5 \sigma$ discovery potential in fluence (time integrated flux) as a function of searched time windows for the max burst search in the northern sky. Right: the $90 \%$ confidence level pre-trial sensitivity and $5 \sigma$ discovery potential for the stacking search as a function of searched time windows in the northern sky. Three signal energy spectra are considered here, $\mathrm{E}^{-2}$ in solid lines, $\mathrm{E}^{-2.5}$ in dashed lines, and $\mathrm{E}^{-3}$ in dot dashed lines.

Two search strategies were carried out based on the test statistic defined in Equation 3.2. One is called the stacking search, which tests the hypotheis that FRBs emit neutrinos as a class of astrophysical sources; the other is called the max burst search, which tests the hypothesis that one or a few FRBs emit neutrinos. In the stacking search, $n_{s}$ and $\left\langle n_{b}\right\rangle$ are the total number of expected signal and background events, respectively, from all FRBs considered. One TS (with its corresponding $n_{s}$ ) value will be returned in one ensemble of events. In the max burst search, $n_{s}$ and $\left\langle n_{b}\right\rangle$ are treated as expected signal and background events, respectively, at a given FRB location, and the $T S$ will be evaluated for each burst with the most optimal one returned in one ensemble of events. The expected background events $\left\langle n_{b}\right\rangle$ are obtained from off time data samples as discussed in Section 2. 
As the potential neutrino arrival time difference with respect to photons is model dependent and unknown, we employed a model independent search using expanding time windows, each of which is centered at the burst times, similar to [14]. A total of 25 time windows were considered, starting from $\Delta T=10 \mathrm{~ms}$ to about 2 days, following an exponential expansion $\Delta T=2^{i} \cdot 10 \mathrm{~ms}$ $(i=0,1, \ldots, 24)$. The various time windows are overlapping, so cross time window trial factors must be accounted for when computing significance values.

\section{Sensitivity and Discovery Potential}
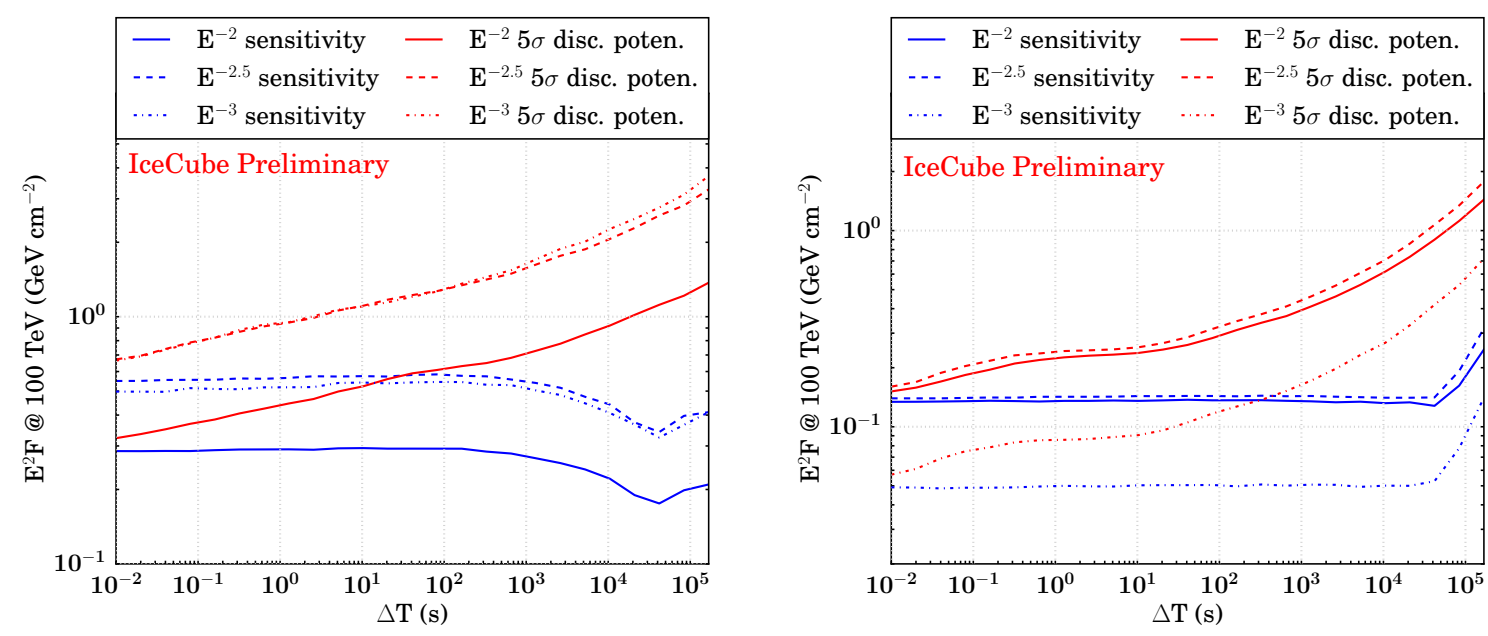

Figure 3: Left: the $90 \%$ confidence level pre-trial sensitivity and $5 \sigma$ discovery potential in fluence (time integrated flux) as a function of searched time windows for the max burst search in the southern sky. Right: the $90 \%$ confidence level pre-trial sensitivity and $5 \sigma$ discovery potential for the stacking search as a function of searched time windows in the southern sky. Three signal energy spectra are considered here, $\mathrm{E}^{-2}$ in solid lines, $\mathrm{E}^{-2.5}$ in dashed lines, and $\mathrm{E}^{-3}$ in dot dashed lines.

Background ensembles were constructed by injecting fake events following a Poissonian distribution with mean $\mu=\left\langle n_{b}\right\rangle$ and event properties that follow their corresponding distributions from the off time event samples. The sensitivity and discovery potential were then calculated by injecting signal events following an assumed energy spectrum on top of injected background events: the injected signal fluence (time integrated flux, denoted as $F$ ) is iterated over until a minimum value is obtained to reach the thresholds set for sensitivity and discovery potential. Here, sensitivity and discovery potential are defined as the minimum signal fluences required to surpass, respectively, the median in $90 \%$ of the trials and $5 \sigma$ point in $90 \%$ of the trials of a background only test statistic distribution. Shown in Fig. 2 and Fig. 3 are the sensitivity and discovery potentials for the northern and southern hemisphere searches, respectively. For both the stacking and max burst searches in both hemispheres, the sensitivity stays flat in the short time windows that are nearly background free. In the longer time windows where background becomes relevant, however, both the sensitivity and discovery potential are rapidly worsening. 


\section{Results}

To avoid artificially introducing a bias for a weak signal search, a blind procedure was employed in this analysis. The on time events were put aside during the analysis development, and only analyzed after unblinding, i.e. after the methods were reviewed and approved by the collaboration. The overall analysis procedure returns the most optimal time window for coincident IceCube events with FRBs and the corresponding pre-trial $p$ values. No significant correlations between neutrinos and FRBs were found. The most significant pre-trial p-value ( $p=0.034)$ among all the searches is in the time window of $655.36 \mathrm{~s}$ of the northern max burst search, and the posttrial p-value is $p=0.25$. The corresponding $T S$ and $n_{s}$ are 3.90 and 0.99 , respectively. The most significant pre-trial p-value for the southern hemisphere is $p=0.412$, which is post-trialled to be $p=0.84$, from the largest time window searched, $167772.16 \mathrm{~s}$. The corresponding $T S$ and $n_{s}$ are 0.64 and 0.78 , respectively. The post-trial p-values were obtained by investigating more background only ensembles. In each such ensemble, a set of expected background events for the largest time window were injected and pre-trial p-values were calculated for each time window with expected events randomly selected from this set of injected events for the specific time window. The most significant pre-trial p-value across all time windows is then selected in each ensemble, and hence form a pre-trial p-value distribution for a set of ensembles. Such a distribution is used to correct for cross time window trial factors. Fig. 4 shows the sky maps of the events that contributed most significantly to their associated nearby FRBs in both the northern and southern max burst searches. The stacking search evaluates all the burst contributions simultaneously so the overall threshold for the scenario that only a few bursts are contributing is higher than the max burst search. The most significant pre-trial p-value for the stacking search in the northern hemisphere is $p=0.074$, corresponding to a post-trial $\mathrm{p}$-value of $p=0.38$, in the same optimal time window of $655.36 \mathrm{~s}$ as the max burst search. The stacking search for the southern hemisphere yielded a null result, i.e. $T S$ and $n_{s}$ equals to zero, across all time windows. The unblinding results are summarized in Table 1.

\section{Conclusion and Outlook}

A search for a high-energy neutrino signal correlated in time and direction with FRBs has been performed, using a maximum likelihood method applied to 6 years of all sky IceCube data. No significant correlation was found for either the northern or southern hemispheres. The most stringent upper limits on neutrino fluence from FRBs for the prompt time window of $\Delta T=10 \mathrm{~ms}$ centered at the burst times are $E^{2} F @ 100 \mathrm{TeV}=0.049 \mathrm{GeVcm}^{-2}$ and $0.134 \mathrm{GeVcm}^{-2}$ for the northern and southern hemispheres, respectively. These limits are much improved, especially in the northern hemisphere, compared to a previous search using only one year of IceCube data which searched only 4 bursts, which also resulted in no correlations [15]. With future radio telescopes with a large instantaneous field of view such as CHIME [16] turning on, on the order of thousands of FRBs per year are expected to be detected. IceCube will continue to follow up on the neutrino correlations with FRBs with similar techniques as discussed in these proceedings. 


\begin{tabular}{|c|c|c|c|c|c|c|}
\hline $\begin{array}{l}\text { Northern } \\
\left(\delta>-5^{\circ}\right)\end{array}$ & $T S$ & $n_{s}$ & $\begin{array}{l}\text { most significant events } \\
\quad\left(\mathrm{t}-\mathrm{t}_{\mathrm{FRB}}, \Delta \Psi\right)\end{array}$ & $\begin{array}{c}\text { pre-trial } p \\
(\text { post-trial } p)\end{array}$ & optimal $\Delta \mathrm{T}$ & coincident FRBs \\
\hline max-burst & 3.90 & 0.99 & $\left(+200.806 \mathrm{~s}, 2.31^{\circ}\right)$ & $\begin{array}{l}0.034 \\
(0.25)\end{array}$ & $655.36 \mathrm{~s}$ & $\begin{array}{c}\text { FRB121102 repeater } \\
\text { 2015/06/02 16:38:07.575 UTC }\end{array}$ \\
\hline stacking & 1.41 & 1.01 & $\left(+200.806 \mathrm{~s}, 2.31^{\circ}\right)$ & $\begin{array}{l}0.074 \\
(0.38)\end{array}$ & $655.36 \mathrm{~s}$ & $\begin{array}{c}\text { FRB121102 repeater } \\
\text { 2015/06/02 16:38:07.575 UTC }\end{array}$ \\
\hline $\begin{array}{l}\text { Southern } \\
\left(\delta<-5^{\circ}\right)\end{array}$ & $T S$ & $n_{s}$ & $\begin{array}{l}\text { most significant events } \\
\quad\left(\mathrm{t}-\mathrm{t}_{\mathrm{FRB}}, \Delta \Psi\right)\end{array}$ & $\begin{array}{c}\text { pre-trial } p \\
(\text { post-trial } p)\end{array}$ & optimal $\Delta \mathrm{T}$ & coincident FRBs \\
\hline max-burst & 0.64 & 0.78 & $\left(-16.9 \mathrm{hrs}, 0.20^{\circ}\right)$ & $\begin{array}{l}0.412 \\
(0.84)\end{array}$ & $167772.16 \mathrm{~s}$ & $\begin{array}{c}\text { FRB 140514 } \\
\text { 2014/05/14 17:14:11.06 UTC }\end{array}$ \\
\hline stacking & 0 & 0 & - & $\begin{array}{c}1.0 \\
(1.0)\end{array}$ & - & - \\
\hline
\end{tabular}

Table 1: Unblinding results for four searches in both the northern and southern hemispheres. Only shown in this table are the most significant bursts with the most significantly contributing IceCube events.
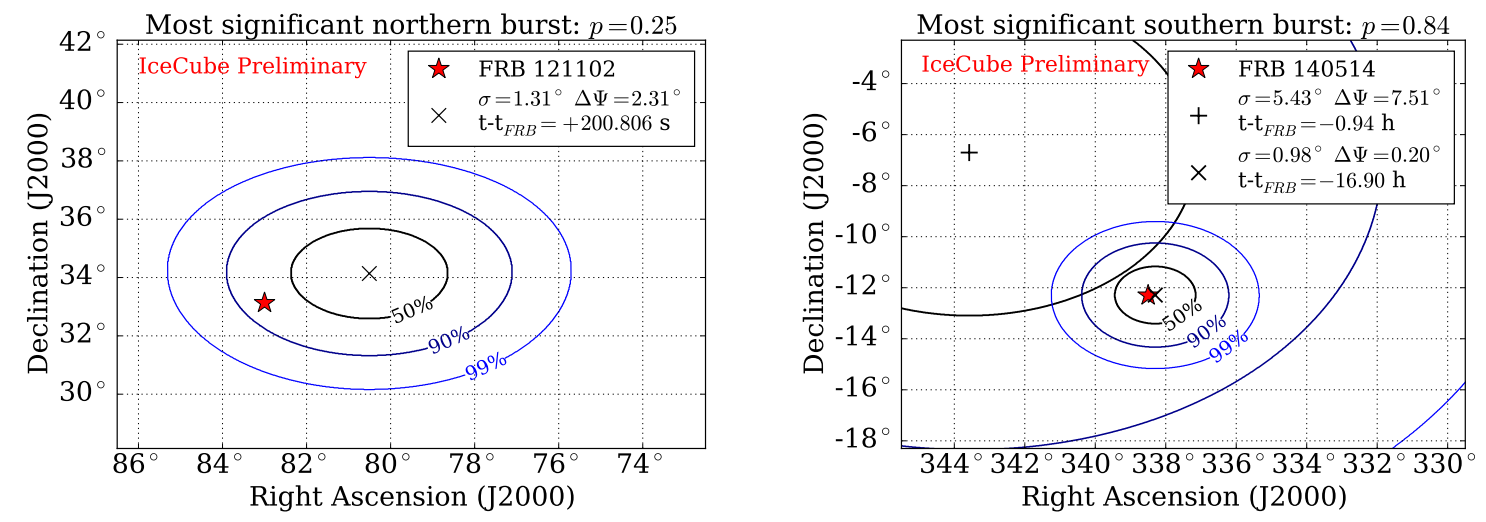

Figure 4: Left: most significant burst and the associated IceCube event from the northern sky max burst search. Right: most significant burst and associated IceCube events from the southern sky max burst search.

\section{References}

[1] D. R. Lorimer, M. Bailes, M. A. McLaughlin, D. J. Narkevic, and F. Crawford, Science 318 (2007) $777-780$.

[2] K. Masui et al., Nature 528 (2015) 523.

[3] L. G. Spitler et al., Astrophys. J. 790 (2014) 101.

[4] P. Scholz et al., Astrophys. J. 833 (2016) 177.

[5] S. Chatterjee et al., Nature 541 (2017) 58. 
[6] D. Thornton, B. Stappers, M. Bailes, B. Barsdell, S. Bates, N. Bhat, M. Burgay, S. Burke-Spolaor, D. Champion, P. Coster, et al., Science 341 (2013) 53-56.

[7] H. Falcke and L. Rezzolla, Astron. Astrophys. 562 (2014) A137.

[8] T. Totani, Publications of the Astronomical Society of Japan 65 (2013) L12.

[9] M. J. REES, Nature 266 (1977) 333-334.

[10] B. D. Metzger, E. Berger, and B. Margalit, Astrophys. J. 841 (2017) 14.

[11] IceCube Collaboration, M. G. Aartsen et al., JINST 12 (2017) P03012.

[12] IceCube Collaboration, M. G. Aartsen et al., The Astrophysical Journal Letters 805 (2015) L5.

[13] IceCube Collaboration, M. G. Aartsen et al., arXiv:1702. 06868.

[14] IceCube Collaboration, R. Abbasi et al., Nature 484 (2012) 351-353.

[15] S. Fahey, A. Kheirandish, J. Vandenbroucke, and D. Xu, arXiv preprint arXiv:1611.03062 (2016).

[16] CHIME Scientific Collaboration, M. Amiri et al., arXiv:1702.08040. 